\title{
Analysis of Rocket Modelling Accuracy and Capsule Landing Safety
}

\author{
Mingyang Huang ${ }^{1}$
}

Received: 14 September 2021 / Revised: 19 December 2021 / Accepted: 20 December 2021 / Published online: 25 January 2022 (c) The Author(s) 2022

\begin{abstract}
The capability of performing vertical landing accurately and safely is of great concern for reusable rockets, some of which might carry manned capsules. According to previous technologies, the rocket needs to accurately land on a drone ship that has a limited size of $70 \mathrm{~m} \times 50 \mathrm{~m}$, whereas the capsule needs to safely land on a soil terrain that might be oblique. Currently, there has been various research supporting the landing processes of rockets and capsules. Regardless of the disparity of such research, the accuracy and safety are the essential indicators to be considered. This paper develops a more accurate landing model for rockets by reviewing valid methodologies for error mitigation. The position error of the rocket (13.46 m) is smaller than the radius of the terminal dock of the drone ship $(25 \mathrm{~m})$; thus, the modelling accuracy of the rocket is within its accuracy requirement (with a 95\% probability). After the performance in terms of accuracy is quantified, a novel landing gear is designed to deal with sloped terrains, which might have a negative effect on landing safety. The struts of the novel landing gear include actuators, buffer struts, and a scanning system. This scanning system is used to extract sloped terrain information, and then the actuators are used for the adjustment of different landing struts, ensuring that these struts touch the sloped terrain at the same time. Compared with conventional landing struts, the novel landing gear is used to increase the buffer efficiencies of the buffer struts.
\end{abstract}

Keywords Analysis $\cdot$ Rocket $\cdot$ Accuracy $\cdot$ Capsule $\cdot$ Safety

\section{Introduction}

Since the 1960s, many countries have carried out experiments on rockets which are used to transport different payloads to the space [1]. Due to the development of space transportation, many companies are struggling on novel rocket technologies to reduce their launch costs [2]. One of the efficient methods of launch cost reduction is to develop reusable rockets, because the multiple uses of such rockets allow the companies to share the manufacture costs [3]. Many private aerospace companies are investing in rockets, which are intended to be retrieved after the launch.

In 2015, Space Exploration Technologies (SpaceX)'s Falcon 9 was an important milestone of the research on reusable rockets [4]. SpaceX announced that using this rocket is able to reduce the launch cost by $80 \%$ [5]. The rocket landed on a drone ship that has a limited size of $70 \mathrm{~m} \times 50 \mathrm{~m}$ [6]. In future, some of these rockets will be used to transport manned cap-

Mingyang Huang

m.huang18@imperial.ac.uk

1 Centre for Transport Studies, Imperial College London, London SW7 2AZ, UK sules, which might land on oblique terrains. Therefore, this paper aims at the dynamic analysis of the rocket and capsule considering the complexity of their landing struts.

In addition to SpaceX's Falcon 9 [7], there have been some other successful examples of the uses of landing struts, for instance, the reusable rocket's landing gear, the near space lander, and the helicopter's landing gear [8]. Most of these landing struts are only suitable for horizontal landing areas. If the landing area is sloped, these struts need to have adjustment functions to adapt to the sloped area.

To adapt to different sloped terrains and achieve their slope landing requirements, seven landing gears with adjustment functions have been developed for different vertical landing vehicles. The first landing gear was designed considering various touchdown situations [9]. The second landing gear was designed for extending the operational range of rotorcrafts [10]. The third landing gear was designed for helicopters [11]. The fourth landing gear was designed for rotorcrafts [12]; however, it is unable to adjust its angle during touchdown [13]. The fifth landing gear was proposed to ensure slope landing [14]. The sixth landing gear was established for landers to satisfy various landing requirements 
[15]. The seventh landing gear is an adaptive landing gear for the sloped landing of launch vehicles and landers [16]. These seven types of landing gears can enhance the usability of different vertical landing vehicles such as rockets; however, they require further analysis to improve the modelling accuracy and landing safety. There have been a number of researchers that analyse the landing dynamics of different vehicles [17-22].

This paper analyses the modelling accuracy of rockets by reviewing valid methodologies for error mitigation. The modelling accuracy of rockets is the basis of landing safety. To meet the safety requirement under different slope landing conditions, a novel landing strut is designed for sloped terrains, as it employs ultrasonic sensors to properly measure the heights of landing struts. According to the deviations of these heights, the actuators are used to rotate the struts, ensuring that all the struts touch the sloped landing surface together. A virtual model is derived for the novel landing strut, and then its dynamic model is established. To properly reflect the performance of the novel landing struts including the peak axial forces and buffer properties, the dynamic analysis is performed. The influences of the novel landing struts on the landing performance are analysed and compared with conventional landing struts.

\section{Rocket Approach Analysis}

Rockets consist of rocket bodies and boosters that are used for the acceleration, and they are combined with payloads such as manned capsules. This paper assumes that the studied rocket and capsule (or integrated second-stage-with-capsule) are both fully reusable.

During a launch process, the rocket continuously uses its engines to accelerate and then vertically moves to a predetermined position with a pre-defined height. After the rocket sends the capsule up to the pre-determined position, the capsule separates from the rocket while the rocket returns to the Earth under its own power. During the return process, the rocket continuously uses its engines to deaccelerate until the time when its engines cut off, and then it approaches and touches down on its landing struts. After the separation process, the capsule floats down on the end of a parachute, which allows recovery of both stages at the launch site. The trajectory profile of the rocket from launch to landing is shown in Fig. 1.

The rocket aims to accurately approach and land on a drone ship, which has a limited size of $70 \mathrm{~m} \times 50 \mathrm{~m}$. The capsule lands on an open terrain, which is much larger than the landing area of the rocket; thus, this section only analyses the modelling accuracy of the rocket, which lands on the drone ship. The prototypes of the rocket and drone ship are shown in Fig. 2.

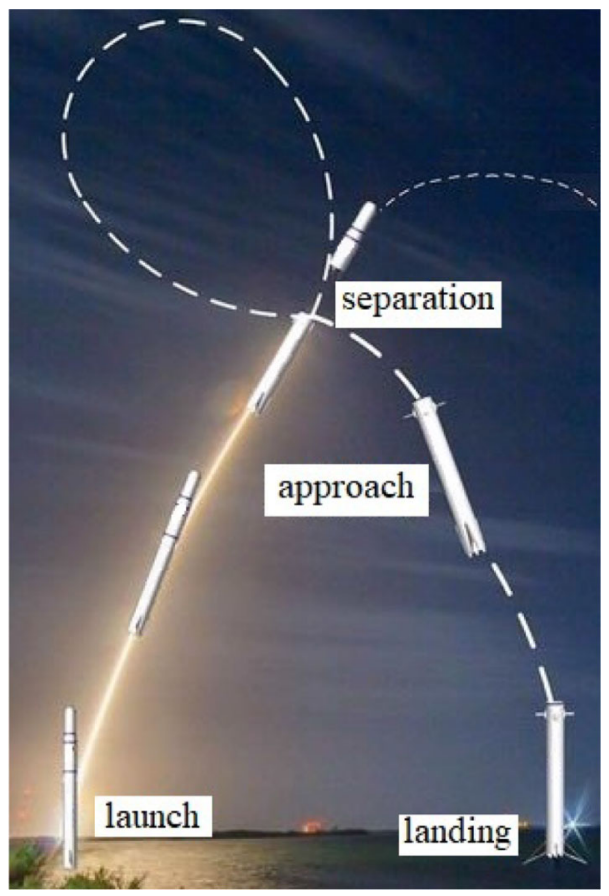

Fig. 1 The trajectory of the rocket from launch to landing

During approach and landing processes, the rocket might have position and other state errors, which have the probability to cause an accident as shown in Fig. 3 .

During the approach process, the modelling accuracy is the essential parameter to be determined; thus, the knowledge of modelling accuracy and its performance evaluation are of paramount importance. Modelling accuracy reflects the capability of the rocket to precisely approach the drone ship. The approach capability is on the basis of the rocket states which are measured by navigation systems. Without an accurate state measurement, modelling accuracy will exceed the upper limit of accuracy requirements and become unacceptable after a short term. There are a number of on-board and off-board navigation systems that have been extensively used for state measurement.

The states of the rocket are represented by a number of state variables, including the position and attitude. For a given rocket, these state variables are collected by various sensors and consolidated into a vector. The state vector consists of at least six state variables: three position variables (i.e. longitude, latitude, altitude) and three attitude variables.

The state variables of the rocket have various errors that are caused by different factors. The position and attitude errors of the rocket on the drone ship are shown in Fig. 4.

The position error is specified in terms of the deviation between the actual and planned positions (or other states) of the rocket, and it is the input of the control instructions of the rocket. The position error is characterised in spatial and temporal dimensions. To mitigate the time error, 

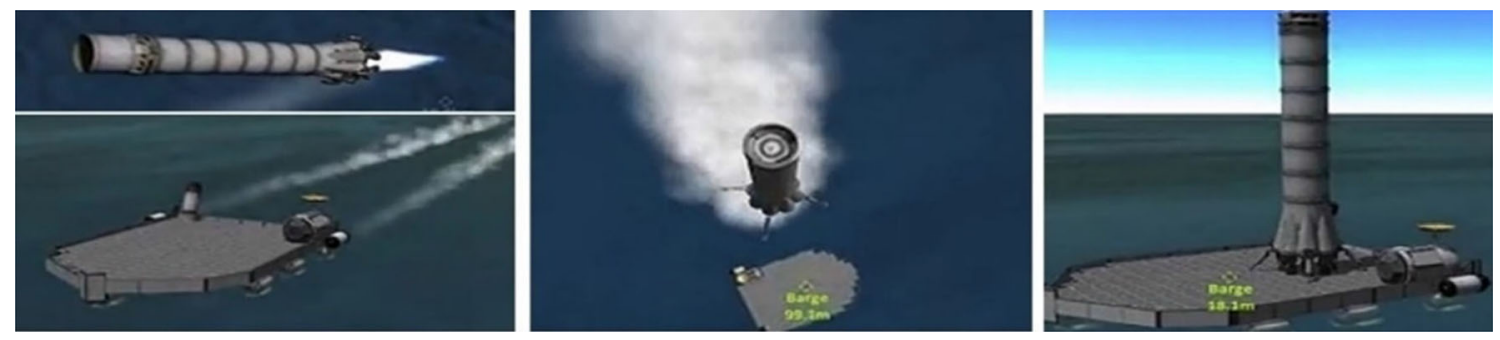

Fig. 2 The prototypes of the rocket and drone ship
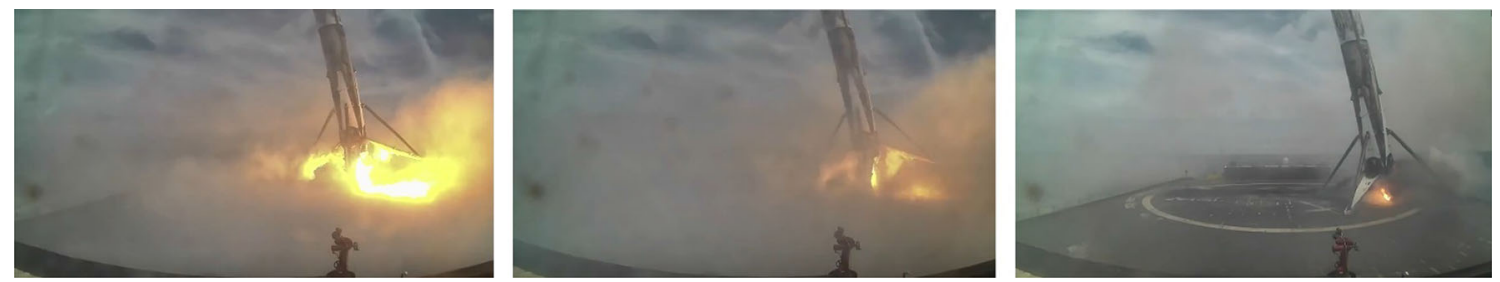

Fig. 3 The accident caused by the position and other state errors of the rocket
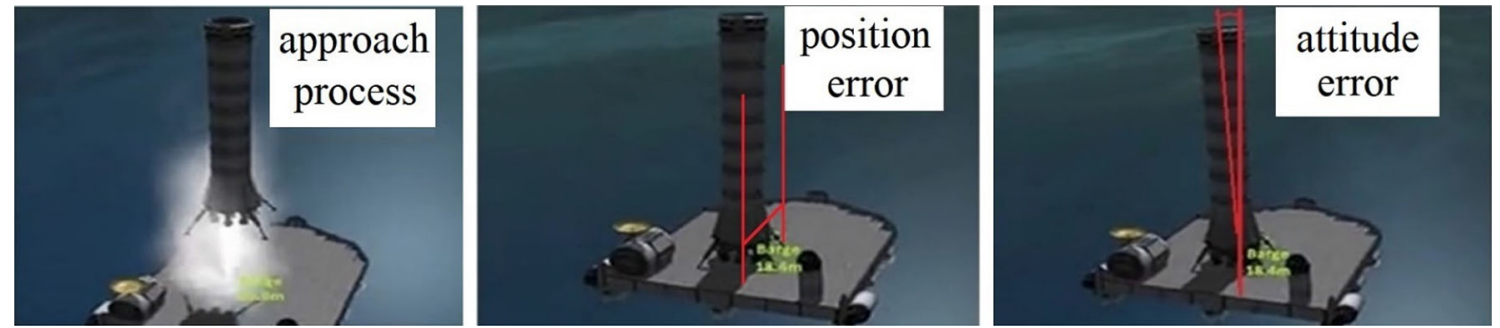

Fig. 4 The position and attitude errors of the rocket

this paper assumes that the rocket maintains an along-track approach during the time interval (i.e. the time error); thus, the time error is transformed into a position error. The position (or other state) error is referred to as a Total System Error (TSE), consisting of a Navigation System Error (NSE) and an Autonomous Approach Error (AAE).

- The NSE is the difference between the actual state and the measured state data obtained by navigation systems, and it reflects the performance of the navigation system. The NSE is caused by navigation systematic errors such as a navigation database coding error in practice.

- The AAE is the error caused by the approach mistakes of the rocket. The rocket performs position recovery abilities once there is an irregular course (e.g. the air disturbance caused by the engine, the position deviation caused by crosswinds). The AAE is influenced by the performance error and wind bias.

The AAE is divided into the performance and weather forecast errors. The TSE is expressed as a function of the NSE, performance error, and weather forecast error in the following equation:
$T S E=$ func (Initial state error, Performance error, Weather forecast error)

The NSE and performance error are assumed as independent and normally distributed with zero mean, and they have collective impacts on TSE. The mathematical model for calculating the TSE is defined in the following equation:

$$
\begin{aligned}
& T S E=\sqrt{N S E^{2}+A A E^{2}} \\
& =\sqrt{N S E^{2}+(\text { Performance error })^{2}+(\text { Weather forecast error })^{2}} .
\end{aligned}
$$

For the NSE, performance error, and weather forecast error, the quantitative analysis is introduced as follows.

\subsection{Navigation System Error}

The states of the rocket are measured by its navigation systems. A typical set of state data usually comprises the initial position and attitude. The measurement accuracy of these initial states strongly influences the modelling accuracy of the rocket. 
The NSE is represented by the deviations between the actual states and their values measured by navigation systems. The navigation system is the backbone (i.e. essential element) of the rocket. To measure the position and attitude, the rocket is equipped with various navigation sensors, including Inertial Measurement Units (IMUs) and Global Navigation Satellite System (GNSS) receivers. The integrated navigation system significantly improves navigation accuracy; however, the GNSS, IMU, and other navigation systems have various error characteristics, which result in different state errors.

\subsubsection{Position Errors}

The position data of the rocket are measured by satellite navigation systems; thus, the position errors are caused by satellite navigation systematic errors.

The position error of the rocket depends on the measurement accuracy of satellite navigation systems. The satellite navigation systems provide position information to different GNSS receivers to support their instantaneous positioning capabilities. The GNSS receivers are used to output the position of the rocket.

Satellite navigation accuracy depends on the satellite-torocket geometry in position measurements. The best method of increasing satellite navigation accuracy is to observe as many satellites as possible, ensuring that the observation directions of the different satellites are in orthogonal directions.

Satellite navigation accuracy depends on a variety of GNSS error sources over a certain period. The GNSS error sources include the satellite (e.g. satellite hardware code, phase delay, satellite clock, satellite orbit, satellite antenna), atmosphere (e.g. ionosphere, troposphere), and receiver (e.g. antenna, receiver clock, receiver hardware delay). Their corresponding errors are introduced in detail as follows:

- The satellite hardware error is caused by different signal components that are processed through the various hardware.

- The satellite signal delay error is caused by the signal delay, which is independent of the satellite geometry.

- The satellite clock error occurs when the satellite clock is not perfectly synchronised with the navigation system time.

- The satellite orbit error is caused by the difference between actual and broadcasted satellite positions.

- The ionosphere error occurs when there is a carrier phase code delay caused by dispersive effects.

- The troposphere error is caused by the signal delay in the neutral atmosphere.

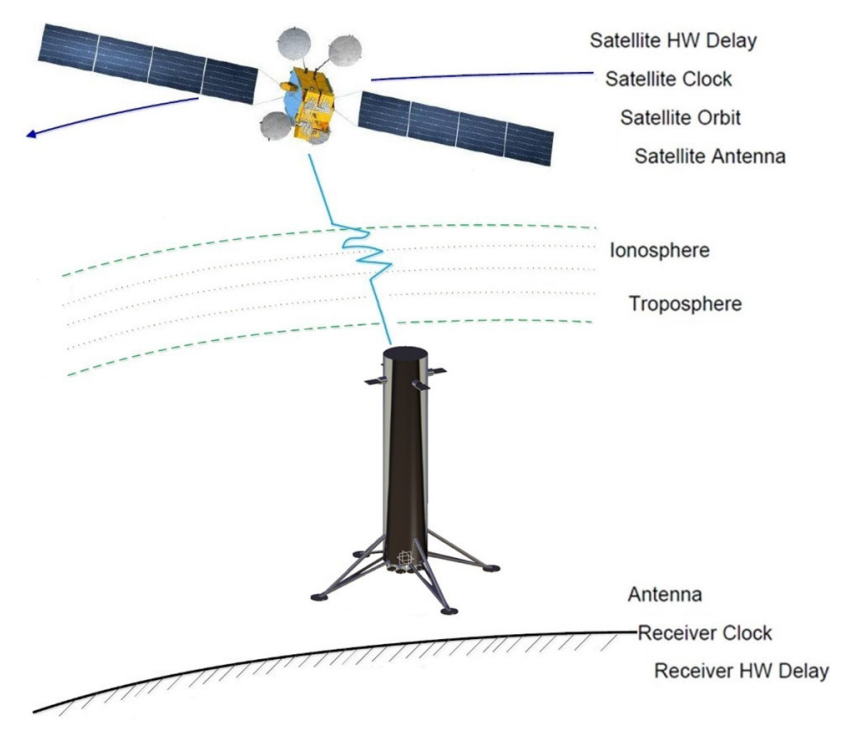

Fig. 5 GNSS error sources

- The receiver clock error occurs when the receiver clock is not perfectly synchronised with the navigation system time.

- The receiver hardware delay error is caused by different signal delays.

The GNSS error sources are shown in Fig. 5.

GNSS errors reflect the satellite navigation accuracy, and they have effects on the position error of the rocket. Due to the artificial degradation of signals, a legacy GNSS signal without any augmentations or differential corrections is generally not of sufficient accuracy for operations. To obtain accurate position measurements, the GNSS errors are mitigated through the technologies such as Real Time Kinematic (RTK) and carrier phase Differential Global Navigation Satellite System (DGNSS). These technologies commonly adopt additional infrastructure (e.g. local base station, reference station, on-board reference unit) to improve satellite navigation accuracy. When integer carrier phase ambiguities are successfully resolved, the carrier phase DGNSS and RTK are able to deliver centimetre-level positioning.

\subsubsection{Attitude Errors}

The attitude data of the rocket are measured by Inertial Navigation Systems (INSs). The INS comprises IMUs and a navigation processing system. The IMU system consists of accelerometers and gyros, which are used to collect the attitude data of the rocket.

The accelerometer and gyro biases grow with time. The INS and GNSS are integrated to mitigate the errors of the sole means of navigation, because their benefits and drawbacks are complementary. The INS is operated continuously with bar hardware faults and low short-term noises. 
The attitude errors of the rocket are forwardly propagated throughout the computation process. To mitigate these errors, GNSS measurements are integrated with the INS to prevent the error propagation. The uptake of the INS has increased with a general advance of GNSS-based solutions and lower prices. The INS is used in poor signal-to-noise environments where satellite visibility is constrained by the environment geometry.

\subsection{Performance Errors}

The performance of the rocket represents the availability of the accurate approach capabilities. The datasets released by their manufacturers return performance information for different input variables on the basis of previous test results.

The performance errors of the rocket endow different error sources, including aerodynamic deterioration and engine performance degradation.

- The aerodynamic performance includes the forces and moments applied on the rocket.

- The engine performance represents the propulsion of the rocket.

Previous performance models only represent the performance of the rocket under specific landing conditions; thus, it is required to introduce the performance degradation in a real-world environment.

\subsubsection{Aerodynamic Errors}

The aerodynamic forces and moments acting over the rocket have strong influences on its states.

Aerodynamic errors endow aerodynamic deterioration which affects aerodynamic characteristics. The deterioration is commonly caused by damaged airframes, deformed aerodynamic surfaces, or missing seals.

Aerodynamic errors pose a great challenge to the rocket due to the lack of aerodynamic data. Considering the complexity of the rocket, the manufacturers may not follow the traditional way in manned aviation to conduct enough tests. Some manufacturers are unwilling to share aerodynamic data which belong to intellectual properties.

The utilisation of rocket data typically leads to a better representation of the actual performance; however, the lack of access to some real-time data results in an inherent error of the performance characteristics of the rocket.

\subsubsection{Propulsion Errors}

The propulsion of the rocket is the thrust force produced by the power plant that is used for deacceleration. The propulsion performance includes the aerodynamic thrust and fuel consumption, which are affected by engine ageing. The fuel consumption is related to the mass, pressure, temperature ratio, and fuel consumption coefficient.

The propulsion error of the rocket is the difference between actual and planned engine thrusts. The actual engine thrust is affected by its flight cycle and pollution.

\subsection{Weather Forecast Errors}

Currently, the weather forecast requires physical models to analytically describe the evolution of weather conditions with time. The main parameters that determine the weather conditions include wind and atmospheric conditions (e.g. temperature, air density, and pressure).

Weather forecast errors are the differences of wind, temperature, air density, and pressure conditions at a given time and position. These errors have influences on modelling accuracy.

The winds acting on the rocket affect its movement through the derivatives of wind vectors with time. The effects of the wind vectors on the rocket movements are determined via wind-generated lift and drag terms.

Wind forecast errors are modelled on the basis of the assumptions of Gaussian distribution and spatial-temporal wind uncertainty correlation. The Computational Fluid Dynamics (CFD) is used to characterise the weather forecast error.

\section{Rocket Approach Error Analysis}

This section reviews the state-of-the-art error mitigation techniques.

\subsection{Navigation System Error Modelling Techniques}

The NSE of the rocket can be mitigated through DGNSS and RTK techniques. It is assumed that the navigation system time is perfectly synchronised with the satellite clock time, and the navigation system is integrated with $5 \mathrm{G}$ wireless systems to deliver centimetre-level positioning. These systems can be used in combination with previous navigation techniques to mitigate the time and position errors to $0 \mathrm{~s}$ and $1 \mathrm{~cm}$.

\subsection{Performance Error Modelling Techniques}

To assess the aerodynamic performance of the rocket, the information from the physics-based models of the rocket is combined with the dynamic sensor data acquired during real landing trials. 


\subsection{Wind Error Modelling Techniques}

On the basis of the 3-D wind information collected by on-board sensors and other weather-sensing systems, the state-of-the-art technologies are able to calculate the 3-D wind force vector to represent varied wind conditions in wind fields.

\subsection{Error Analysis}

The rocket is able to maintain its real-time position to mitigate its state errors. The error sources of modelling accuracy and their corresponding error mitigation have been consolidated into an exhaustive list in Table 1.

Modelling accuracy is expressed as the TSE of the rocket. The NSE (lateral error $2 \mathrm{~cm}$, longitudinal error $2 \mathrm{~cm}$, altitude error $3 \mathrm{~cm})$, atmosphere forecast error $(2 \mathrm{~m})$ and wind forecast error $(2 \mathrm{~m})$ are considered in the calculation of the TSE. A more accurate approach corresponds to a smaller tolerable TSE; thus, the achievable accuracy requirement is analysed by reviewing the error components of the rocket.

It is assumed that the distribution of each error component (denoted by $\mid$ Error component $\mid$ ) is a normal distribution. The probability of the actual magnitude of each error component is expressed as $\frac{1}{\sqrt{2 \pi} \sigma_{\text {Error component }}} e^{\frac{\mid \text { Error component }\left.\right|^{2}}{2 \sigma_{\text {Error component }}^{2}}}$, where $e$ represents Euler Number and $\sigma_{\text {Error component }}$ represents the standard deviation of the error component. The actual magnitude of each error component needs to be within a specified performance limit (denoted by $\pm 2 \sigma_{\text {Error component) for } 95 \% \text { of }}$ the time.

The standard deviation of the total error after the process of error mitigation is denoted by $\sigma_{\text {Total error }}$. The mathematical model for calculating the residual magnitude of the TSE after the process of error mitigation (denoted by $T S E_{\text {mitigation }}$ ) is expressed in the following equation: of the drone ship (i.e. $25 \mathrm{~m})$. The TSE $(13.46 \mathrm{~m})$ is smaller than $25 \mathrm{~m}$; thus, the modelling accuracy of the rocket is within its accuracy requirement (with a 95\% probability). If the position error of the rocket exceeds this requirement, a warning should be issued from its on-board systems, directing position and attitude adjustment to avoid potential threats of landing safety.

The landing safety of the rocket is correlated to not only the accuracy of its terminal operation but also the landing area (Fig. 6); thus, the landing safety needs further analysis considering the landing area in the following sections.

\section{Rocket and Capsule Landing Analysis}

The landing process is the key phase for not only the rocket but also the payload (i.e. capsule). The touchdown determines the success of the whole process of landing. The centre-ofgravity of the rocket is high; thus, it is likely to roll, tilt, and fall over at touchdown. The loss of safety of the rocket caused a landing accident on the drone ship as shown in Fig. 7, where the rocket is awkwardly tilted in an unstable direction.

For safety considerations, the rocket is ordered to land on the drone ship to avoid the influence on its surroundings, whereas the capsule can land on different areas due to its relatively smaller size. Compared with the landing area (i.e. the drone ship) of the rocket, the landing area of the capsule is larger; thus, the capsule does not need to land on a plot with metre-level accuracy. The capsule can only land on a soft terrain when its landing struts are folded, whereas it is able to extend its landing struts to ensure the safe touchdown on a hard terrain as shown in Fig. 8.

The capsule has a probability to land on a sloped terrain; thus, a series of novel landing gears are required for the capsule (and the rocket) to increase the landing safety on

$$
\begin{aligned}
T S & E_{\text {mitigation }}=4 \sigma_{\text {Total error }}+(\text { rocket radius })+(\text { landing strut length }) \\
& =4 \sqrt{\sigma_{\text {Initial state error }}^{2}+\sigma_{\mathrm{UAV}}^{2} \text { performance error }}+\sigma_{\text {Weather forecast error }}^{2}+(\text { rocket radius })+(\text { landing strut length }) \\
& =2.83 \mathrm{~m}+1.83 \mathrm{~m}+8.8 \mathrm{~m} \\
& =13.46 \mathrm{~m} .
\end{aligned}
$$

According to Eq. (3), the statistic characteristic of the errors is $2.83 \mathrm{~m}$ for the terminal operation. The radius of the rocket is $1.83 \mathrm{~m}$ after its landing struts are folded, and the length of the landing strut is $\mathrm{m}$; thus, the TSE is $13.46 \mathrm{~m}$ (with a 95\% probability).

The total error is compared with the accuracy requirement, which is determined as the radius of the terminal dock different sloped terrains. The novel landing struts adopt the conventional landing strut configuration as the basic framework for actuation.

For vertical landing vehicles, the conventional landing systems include four fundamental parts: main struts, buffer struts, actuators, and skid plates. The buffer strut extends outward after it is driven by the actuator. Before the landing 


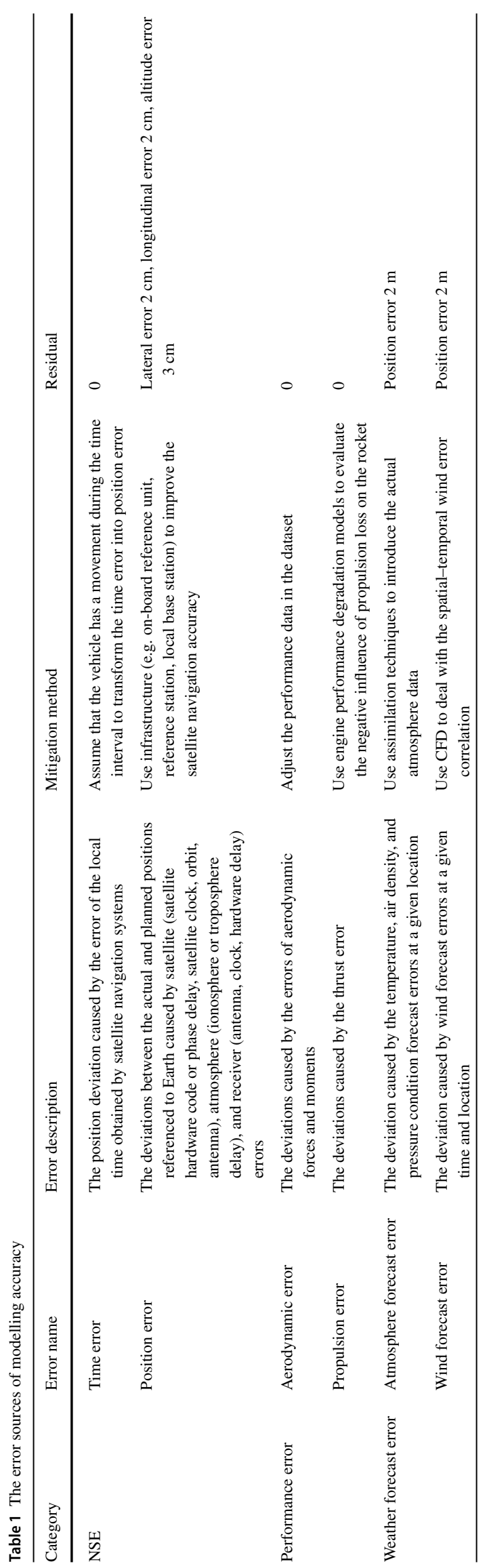

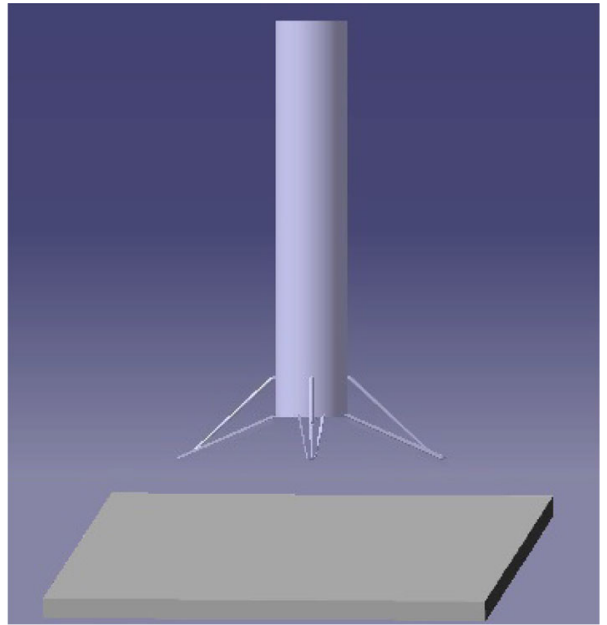

Fig. 6 The landing area of the rocket

process, the main struts are first lowered due to their gravities. At touchdown, the buffer struts are briefly compressed, and then they re-bounce back to their stable shapes. The novel landing struts are also divided into main struts, buffer struts, actuators, and skid plates.

The capsule is equipped with the novel landing struts replacing the conventional landing struts. Each landing strut consists of a main strut, a buffer strut, an actuator, and a skid plate. The virtual prototype of the capsule has been established, and the overall scheme of the capsule equipped with the novel landing struts is shown in Fig. 9.

The axes of the buffer strut and actuator are in the same direction. The buffer strut contains a shock absorber absorbing the landing energy along its axis. The overall scheme of the buffer strut and actuator is shown in Fig. 10.

The whole process of slope landing is shown in Fig. 11.

The usability and validity of the novel landing struts have been demonstrated using drones to replace capsules as shown in Fig. 12.

The utilisation of the novel landing struts can also be expanded to rockets landing on dynamic sloped areas (e.g. rolling drone ships at sea) as shown in Fig. 13.

The logic diagram of the novel landing struts is shown in Fig. 14.

\section{Landing Safety Analysis}

This section establishes the dynamic model of the novel landing struts to analyse their landing performance, including the peak axial forces and buffer properties.

\subsection{Coordinate Systems}

Before the creation of the vehicle dynamic landing model, three right-handed coordinate systems are defined: ground 

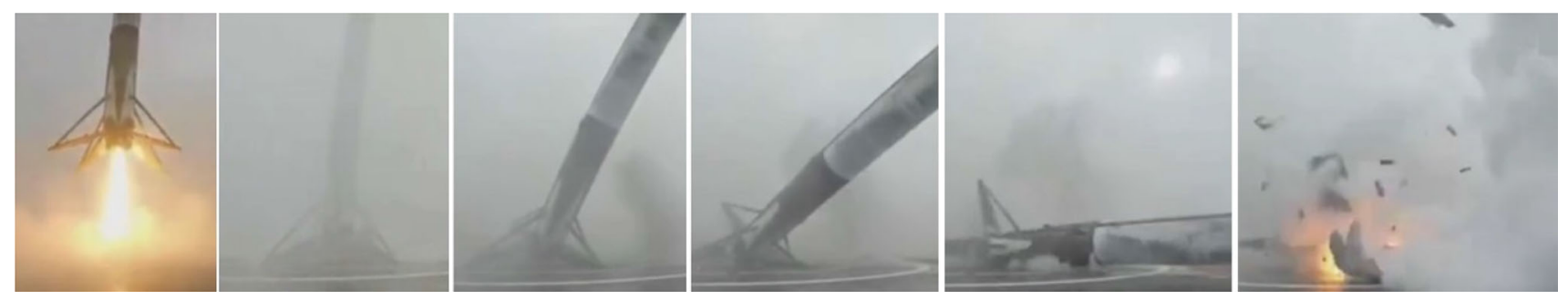

Fig. 7 The loss of safety of the rocket

Fig. 8 Different landing areas

Fig. 9 The overall scheme of the capsule equipped with the novel landing struts

Fig. 10 The overall scheme of the buffer strut and actuator
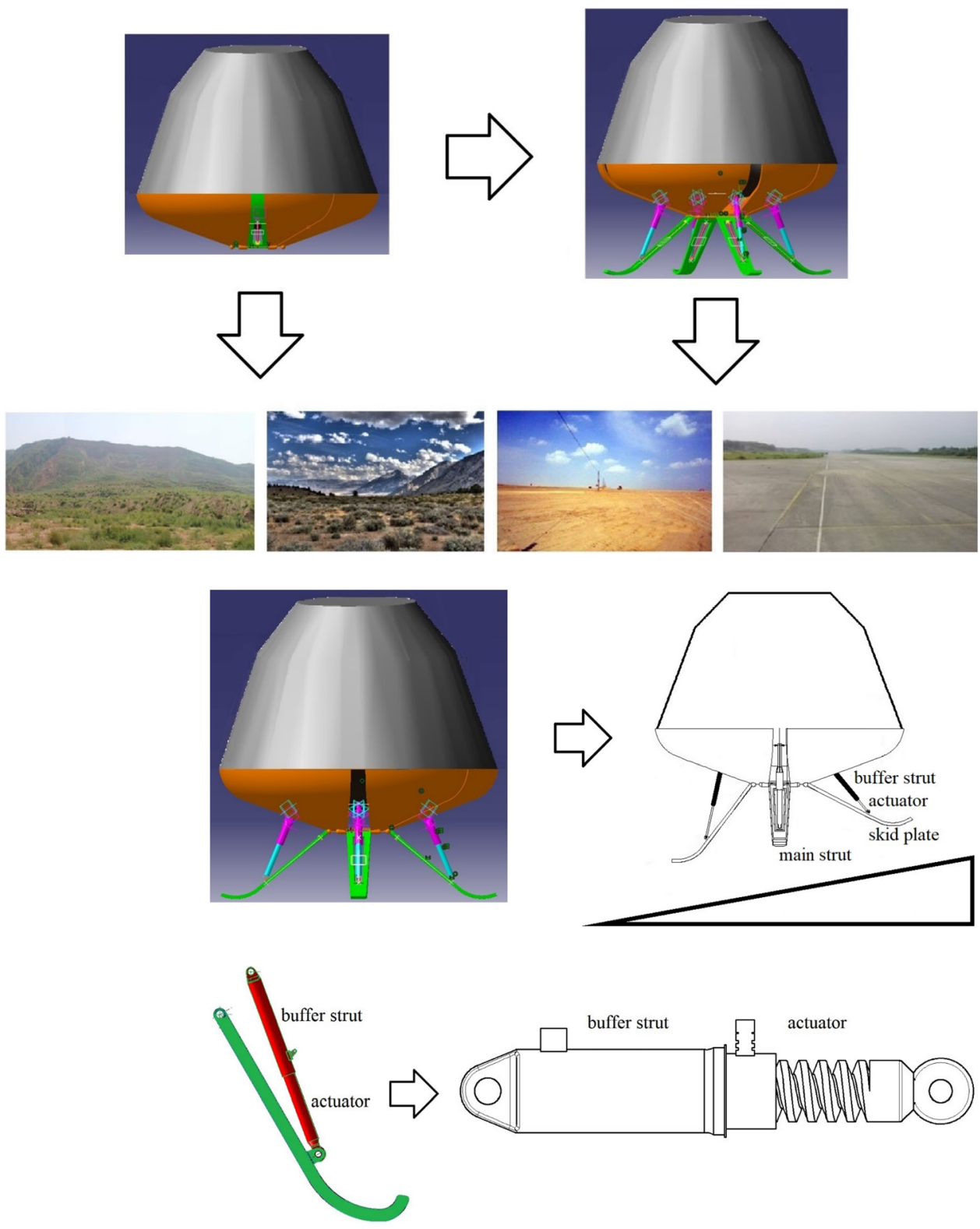
Fig. 11 The slope landing process

Fig. 12 The novel landing struts demonstrated using drones

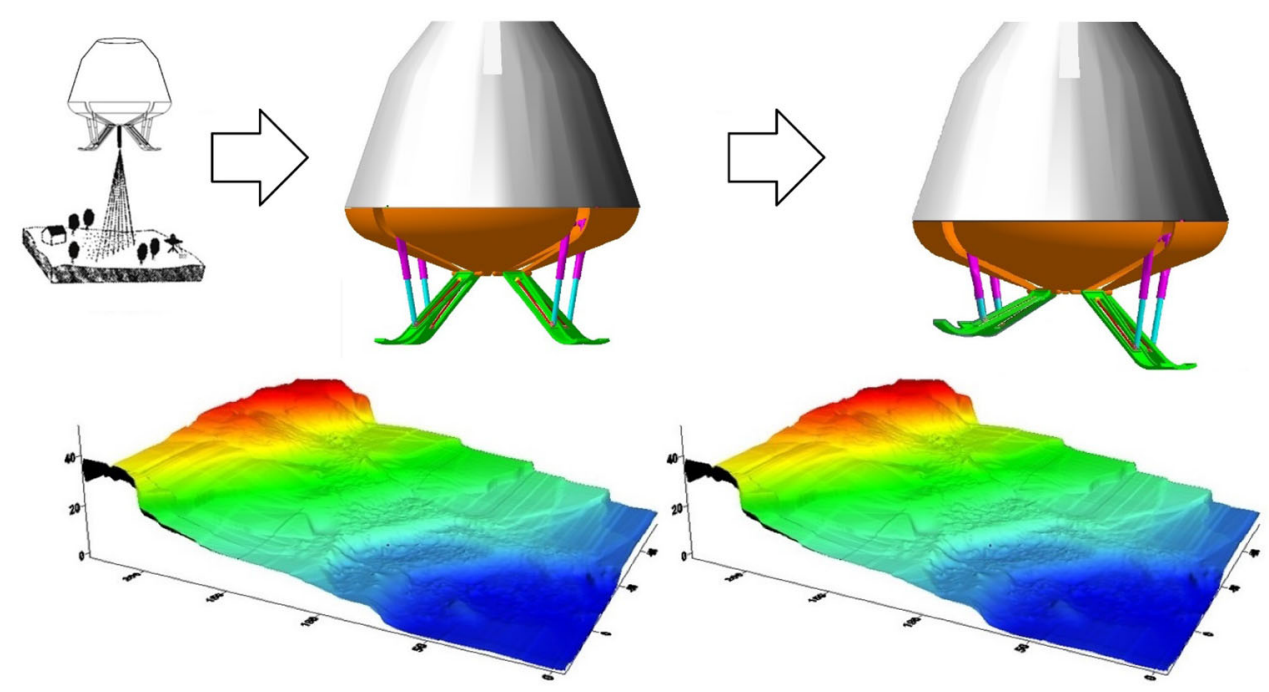

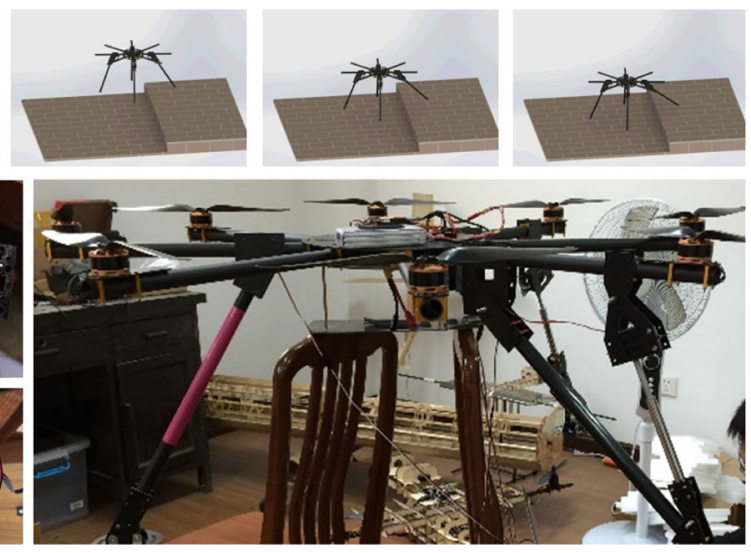

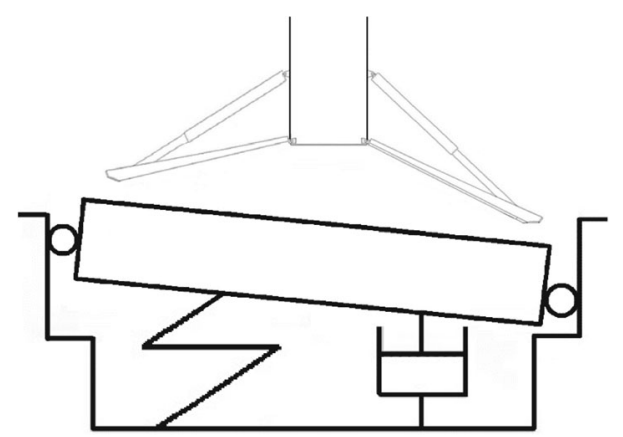

Fig. 13 The novel landing struts utilised on a rolling drone ship

coordinate system $O_{g}-X_{g} Y_{g} Z_{g}$, body coordinate system $O_{b}-X_{b} Y_{b} Z_{b}$, and $i$ th main strut coordinate system $O_{s i}-$ $X_{s i} Y_{s i} Z_{s i}$.

Representation with Euler angles of the two frames: $\theta$ is the pitch angle; $\psi$ is the yaw angle; and $\phi$ is the roll angle. The conversion between body coordinate system and ground coordinate system is described via the matrix $\mathbf{M}_{\mathrm{gb}}$ :

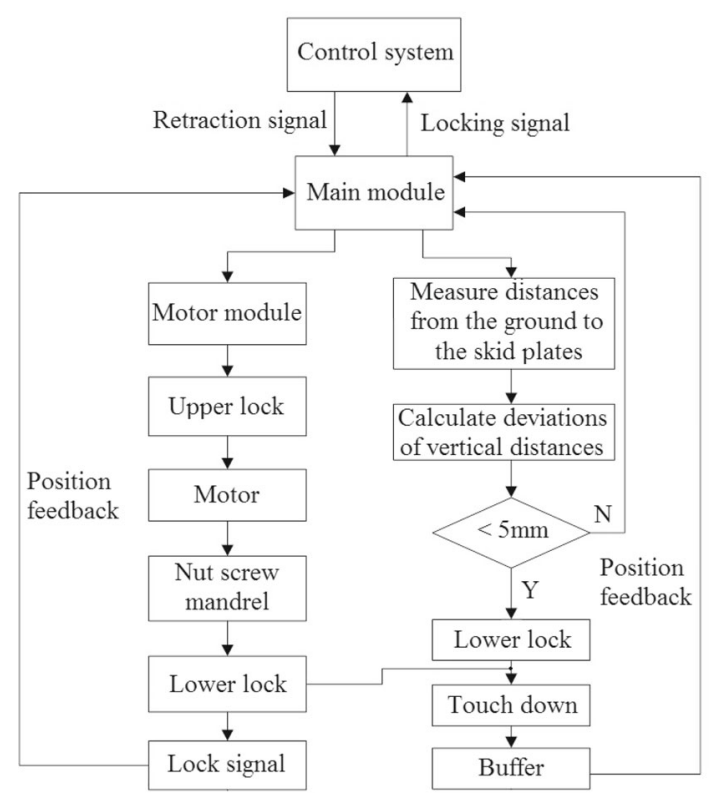

Fig. 14 The logic diagram of the novel landing struts 
Fig. 15 The ground and body coordinate systems

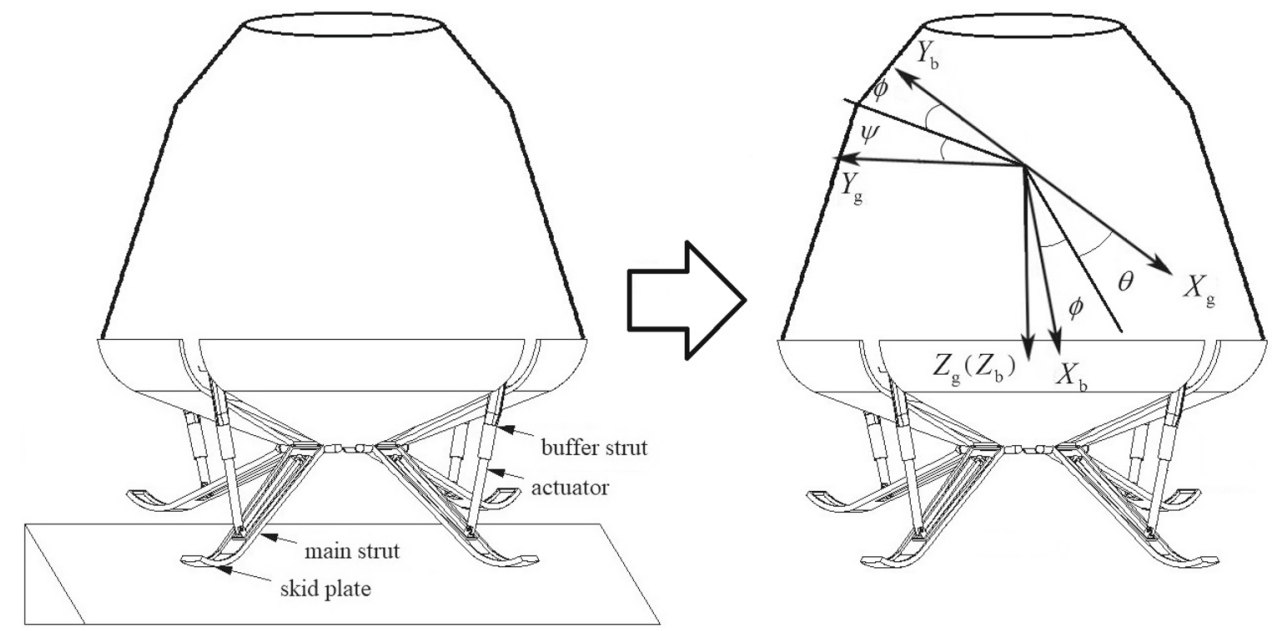

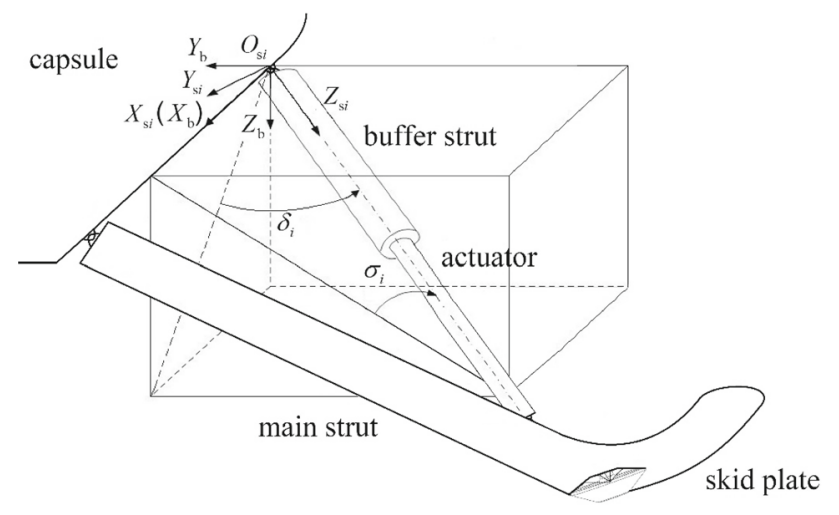

Fig. 16 The relationship between the body and strut coordinate systems

\subsection{Dynamic Equations of Elastic Mass}

The translational equation of elastic mass (i.e. all the parts and components upper the intermediate gasket) in $O_{g}-X_{g} Y_{g} Z_{g}$ is denoted as follows:

$$
\begin{aligned}
m_{b}\left[\begin{array}{c}
\ddot{x}_{b} \\
\ddot{y}_{b} \\
\ddot{z}_{b}
\end{array}\right]= & \mathbf{M}_{\mathrm{gb}}\left\{-\sum_{i=1}^{4} \mathbf{M}_{\mathrm{sib}}^{-1}\left[\begin{array}{c}
F_{x i} \\
F_{y i} \\
F_{z i}
\end{array}\right]-\left[\begin{array}{c}
D \\
-C \\
L
\end{array}\right]\right\} \\
& +\left[\begin{array}{c}
0 \\
0 \\
W_{\mathrm{b}}
\end{array}\right] .
\end{aligned}
$$

$$
\begin{aligned}
& \mathbf{M}_{\mathrm{gb}}=\mathbf{M}_{x}(\phi) \mathbf{M}_{y}(\theta) \mathbf{M}_{z}(\psi) \\
& =\left[\begin{array}{ccc}
\cos \theta \cos \psi & \sin \phi \sin \theta \cos \psi-\cos \phi \sin \psi & \cos \phi \sin \theta \cos \psi+\sin \phi \sin \psi \\
\cos \theta \sin \psi & \sin \phi \sin \theta \sin \psi+\cos \phi \cos \psi & \cos \phi \sin \theta \sin \psi-\sin \phi \cos \psi \\
-\sin \theta & \sin \phi \cos \theta & \cos \phi \cos \theta
\end{array}\right] .
\end{aligned}
$$

The ground and body coordinate systems are shown in Fig. 15.

The matrix $\mathbf{M}_{\mathrm{sib}}$ is the transformation from the body coordinate system to the $i$ th strut coordinate system, where $\sigma_{i}$ and $\delta_{i}$ are the camber angles of the $i$ th strut coordinate system relative to the $X_{\mathrm{b}}-$ axis and $Y_{b}$ - axis of the body coordinate system:

$$
\begin{aligned}
\mathbf{M}_{\mathrm{sib}} & =\mathbf{M}\left(\delta_{i}\right) \mathbf{M}\left(\sigma_{i}\right) \\
& =\left[\begin{array}{ccc}
\cos \sigma_{i} & 0 & -\sin \sigma_{i} \\
-\sin \sigma_{i} \sin \delta_{i} & \cos \delta_{i} & -\cos \sigma_{i} \sin \delta_{i} \\
\sin \sigma_{i} \cos \delta_{i} & \sin \delta_{i} & \cos \sigma_{i} \cos \delta_{i}
\end{array}\right] .
\end{aligned}
$$

The $i$ th strut coordinate system and body coordinate system are shown in Fig. 16.
The rotation equation of elastic mass in $O_{b}-X_{b} Y_{b} Z_{b}$ is denoted as follows:

$$
\begin{aligned}
\mathbf{I}_{\mathrm{b}}\left[\begin{array}{c}
\dot{\omega}_{x} \\
\dot{\omega}_{y} \\
\dot{\omega}_{z}
\end{array}\right]= & -\left[\begin{array}{ccc}
0 & -\omega_{z} & \omega_{y} \\
\omega_{z} & 0 & -\omega_{x} \\
-\omega_{y} & \omega_{x} & 0
\end{array}\right] \mathbf{I}_{\mathrm{b}}\left[\begin{array}{c}
\omega_{x} \\
\omega_{y} \\
\omega_{z}
\end{array}\right] \\
& +\sum_{i=1}^{4} \mathbf{M}_{\mathrm{s} i \mathrm{~b}}^{-1}\left[\begin{array}{c}
T_{x i} \\
T_{y i} \\
T_{z i}
\end{array}\right] \\
& +\sum_{i=1}^{4}\left[\begin{array}{ccc}
0 & -z_{\mathrm{s} i} & y_{\mathrm{si}} \\
z_{\mathrm{s} i} & 0 & -x_{\mathrm{s} i} \\
-y_{\mathrm{s} i} & x_{\mathrm{s} i} & 0
\end{array}\right] \mathbf{M}_{\mathrm{sib}}^{-1}\left[\begin{array}{c}
F_{x i} \\
F_{y i} \\
F_{z i}
\end{array}\right] \\
& -\left[\begin{array}{ccc}
0 & -z_{c} & y_{l} \\
z_{d} & 0 & -x_{l} \\
-y_{d} & x_{c} & 0
\end{array}\right]\left[\begin{array}{c}
D \\
-C \\
L
\end{array}\right],
\end{aligned}
$$


where $\omega_{x}, \omega_{y}, \omega_{z}$ are the angular speeds of elastic mass along $O_{\mathrm{b}}-X_{\mathrm{b}}$ direction, $O_{\mathrm{b}}-Y_{\mathrm{b}}$ direction and $O_{\mathrm{b}}-Z_{\mathrm{b}}$ direction; $m_{b}$ and $\mathbf{I}_{\mathrm{b}}$ are the mass and the moment of inertia of the body, respectively; $\left[\ddot{x}_{b}, \ddot{y}_{b}, \ddot{z}_{b}\right]^{T}$ is the acceleration value of centre-of-gravity of the body; $\left[0,0, W_{\mathrm{b}}\right]^{T}$ is the gravity force vector of the body; $\left[F_{x i}, F_{y i}, F_{z i}\right]^{T}$ and $\left[T_{x i}, T_{y i}, T_{z i}\right]^{T}$ are the force vector and torque from the $i$ th main strut to the body; $[D,-C, L]^{T}$ is the force vector applied on the body by rocket engines; $x_{\mathrm{s} i}, y_{\mathrm{s} i}, z_{\mathrm{s} i}$ are the distances from the vehicle centre-of-gravity to the $i$ th junction point along $O_{\mathrm{b}}-X_{\mathrm{b}}$ direction, $O_{\mathrm{b}}-Y_{\mathrm{b}}$ direction and $O_{\mathrm{b}}-Z_{\mathrm{b}}$ direction.

$\mathbf{I}_{\mathrm{b}}$ is expressed as follows:

$\mathbf{I}_{\mathrm{b}}=\left[\begin{array}{ccc}I_{x x} & -I_{x y} & -I_{x z} \\ -I_{y x} & I_{y y} & -I_{y z} \\ -I_{z x} & -I_{z y} & I_{z z}\end{array}\right]$

The relationship between body angular rates and Euler angular rates is denoted as follows:

$\left[\begin{array}{c}\dot{\theta} \\ \dot{\phi} \\ \dot{\varphi}\end{array}\right]=\left[\begin{array}{ccc}\cos \phi \cdot \cos \varphi & \sin \varphi & 0 \\ -\cos \phi \cdot \sin \varphi & \cos \varphi & 0 \\ \sin \phi & 0 & 1\end{array}\right]\left[\begin{array}{l}\omega_{x} \\ \omega_{y} \\ \omega_{z}\end{array}\right]$.

\subsection{Dynamic Equations of Inelastic Mass}

The inelastic mass consists of the skid plate and piston under the intermediate gasket. The translational equation of $i$ th inelastic mass in $O_{s i}-X_{s i} Y_{s i} Z_{s i}$ is denoted as follows:

$m_{i}\left[\begin{array}{c}\ddot{x}_{\mathrm{s} i} \\ \ddot{y}_{\mathrm{s} i} \\ \ddot{z}_{\mathrm{s} i}\end{array}\right]=\mathbf{M}_{\mathrm{gb}} \mathbf{M}_{\mathrm{s} i \mathrm{~b}}^{-1}\left[\begin{array}{c}F_{\mathrm{x} i} \\ F_{\mathrm{y} i} \\ F_{\mathrm{z} i}\end{array}\right]+\sum_{i=1}^{4} \mathbf{M}_{\mathrm{gb}}\left[\begin{array}{c}F_{\mathrm{d} i} \\ F_{\mathrm{c} i} \\ F_{\mathrm{n} i}\end{array}\right]+\left[\begin{array}{c}0 \\ 0 \\ W_{i}\end{array}\right]$,

where $m_{i}$ is the $i$ th inelastic support mass; $\left[0,0, W_{i}\right]^{T}$ is the gravity force vector of the $i$ th inelastic mass; $[\ddot{x}, \ddot{y}, \ddot{z}]^{T}$ is the acceleration value of the whole vehicle in ground coordinate system; $\left[F_{\mathrm{d} i}, F_{\mathrm{c} i}, F_{\mathrm{n} i}\right]^{T}$ are the ground reaction forces on the $i$ th skid plate, which is transmitted from the ground to the $i$ th landing strut.

\subsection{Dynamic Equations of Buffer Strut}

The buffer strut includes an outer cylinder, piston, separator and orifices. The force from the $i$ th main strut to the fuselage in $O_{s i}-X_{s i} Y_{s i} Z_{s i}$ is denoted as follows:

$$
\left[\begin{array}{c}
F_{x i} \\
F_{y i} \\
F_{z i}
\end{array}\right]=\left[\begin{array}{c}
K_{x i} \Delta x_{i} \\
K_{y i} \Delta y_{i} \\
F_{\mathrm{h} i}+F_{\mathrm{a} i}+F_{\mathrm{f} i}
\end{array}\right]
$$

where $K_{x i}$ and $K_{y i}$ are the stiffness of the $i$ th main strut along $O_{\mathrm{s} i}-X_{\mathrm{s} i}$ direction and along $O_{\mathrm{s} i}-Y_{\mathrm{s} i}$ direction; $\Delta x_{i}$ and $\Delta y_{i}$ are the elastic deformations of the $i$ th main strut along $O_{\mathrm{s} i}-X_{\mathrm{s} i}$ direction and along $O_{\mathrm{s} i}-Y_{\mathrm{s} i}$ direction; the axial force (denoted by $F_{z i}$ ) of the $i$ th buffer strut's buffer strut consists of oil damping force $F_{\mathrm{h} i}$, air spring force $F_{\mathrm{a} i}$ and inner friction force $F_{\mathrm{f} i}$.

\section{Dynamic Analysis of the Novel Landing Struts}

The dynamic analysis of the novel landing struts is implemented considering two landing conditions. The rocket lands on a hard drone ship, whereas the capsule containing passengers, astronauts, or precision instrument lands on a soil terrain.

The dynamic analysis is performed for comparison between the novel landing struts and conventional landing struts. When landing on a sloped terrain, the four struts of the conventional landing struts touch the ground at different time, whereas the four struts the novel landing struts touch the oblique terrain together. The dynamic analysis of the novel landing struts is shown in Fig. 17.

The dynamic analysis is established considering the flexibility of the landing struts. In the simulation, the flexible landing struts are built and integrated into a rigid-flexible coupling model. The comparison of the axial forces of the buffer struts between the novel landing struts and conventional landing struts is shown in Fig. 18.

The vibrations of the curves are caused by the flexibility of the landing struts in the rigid-flexible coupling model. Each of the axial forces of the buffer struts has two peaks, which occur at the moment of first touchdown and the end of the buffer process. The peak axial forces of the buffer struts mainly occur from 0.05 to $0.2 \mathrm{~s}$.

The variation of length of the buffer strut reflects its compression performance. The buffer strut axial force-stroke curves of the novel landing struts and conventional landing struts are shown in Fig. 19.

Figures 18a and 19a show that the most compressed buffer strut of the conventional landing gear has a low efficiency that is less than $65 \%$ during the landing process. Figure $18 \mathrm{~b}$ and $19 \mathrm{~b}$ illustrate that the buffer efficiencies of the novel landing struts are all more than $85 \%$.

The dynamic analysis of the capsule is shown in Fig. 20.

When landing on a sloped terrain, the four struts of the conventional landing struts touch the ground at different time, whereas the four struts of the novel landing struts touch the oblique terrain together. For the capsule, the buffer strut axial force-stroke curves of the novel landing struts and conventional landing struts are shown in Fig. 21. 
Fig. 17 The dynamic analysis of the novel landing struts
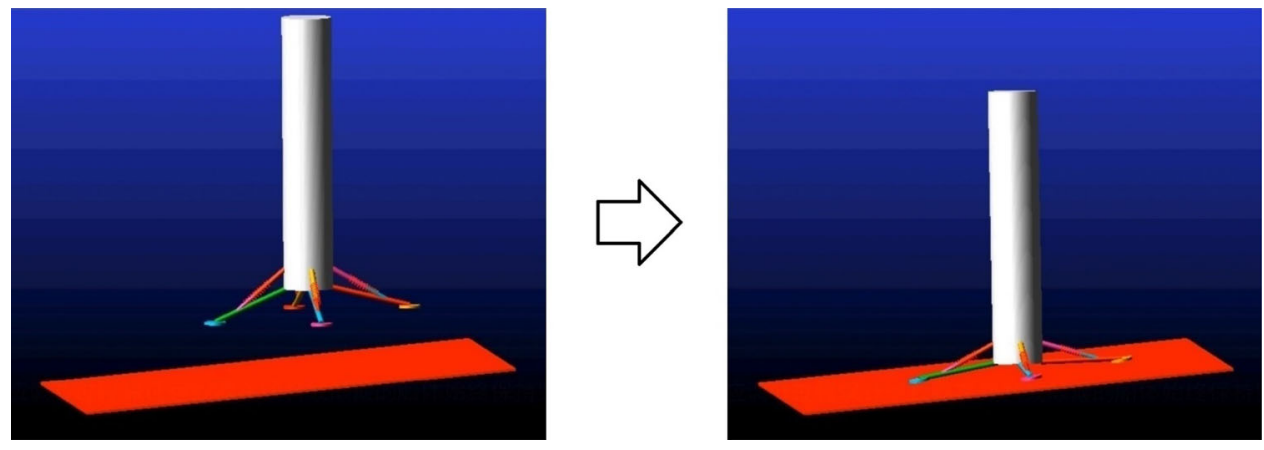

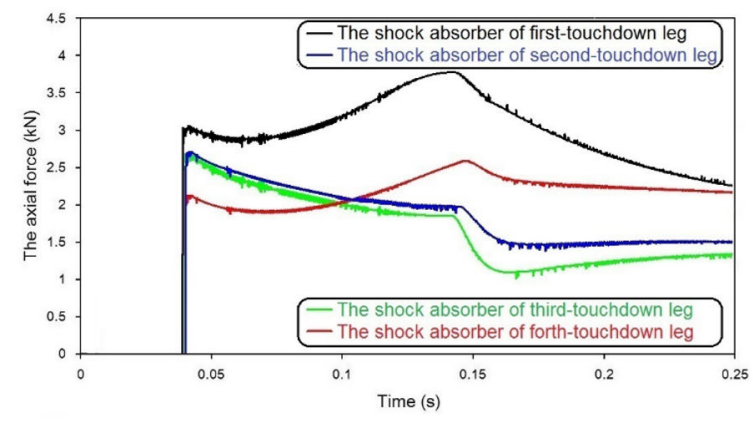

(a) Conventional landing struts

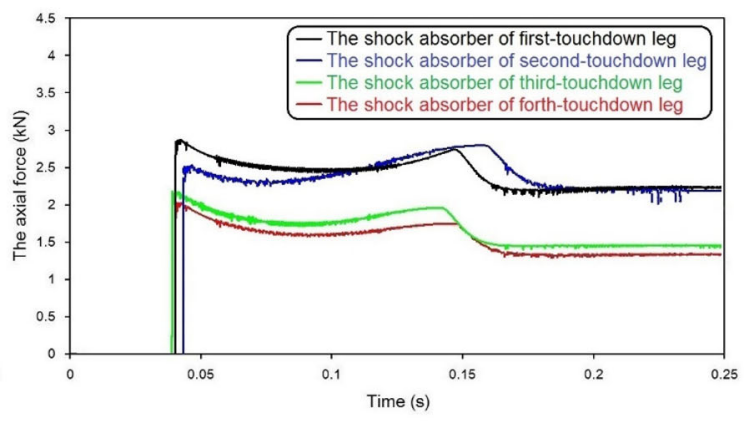

(b) The novel landing struts

Fig. 18 The axial forces of buffer struts

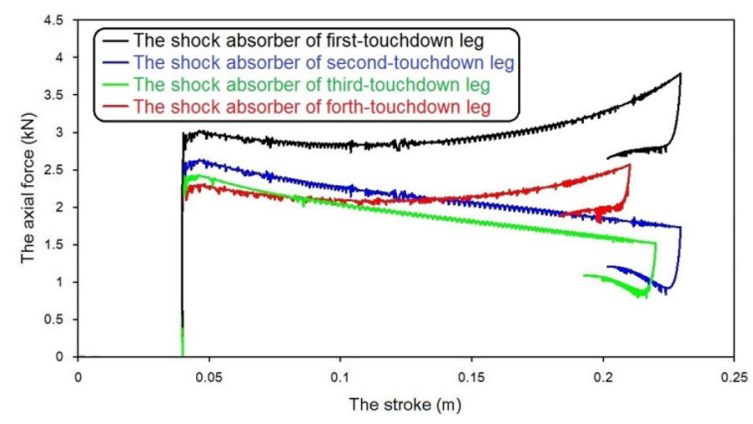

(a) Conventional struts

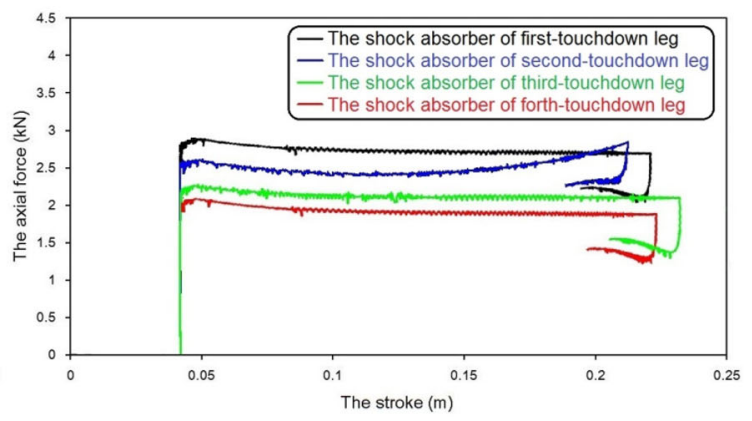

(b) Novel struts

Fig. 19 The axial force-stroke curves of buffer struts

Fig. 20 The dynamic analysis of the capsule
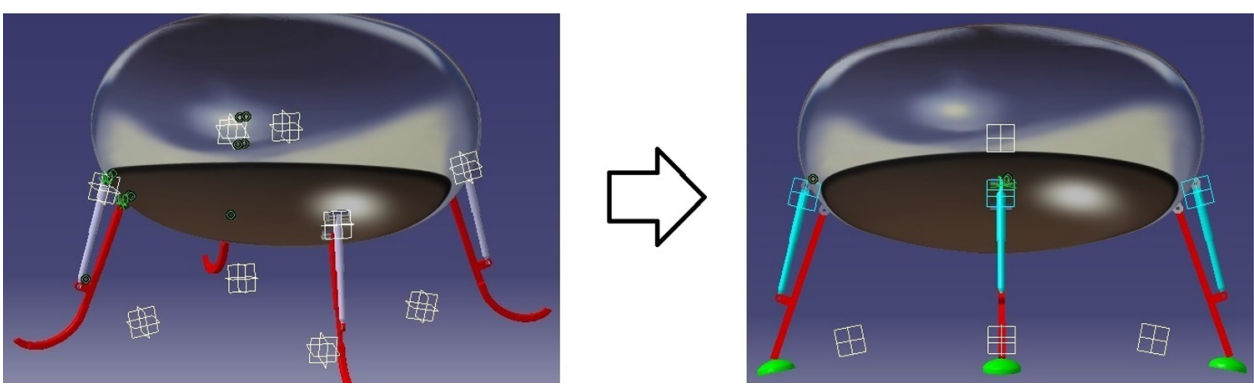


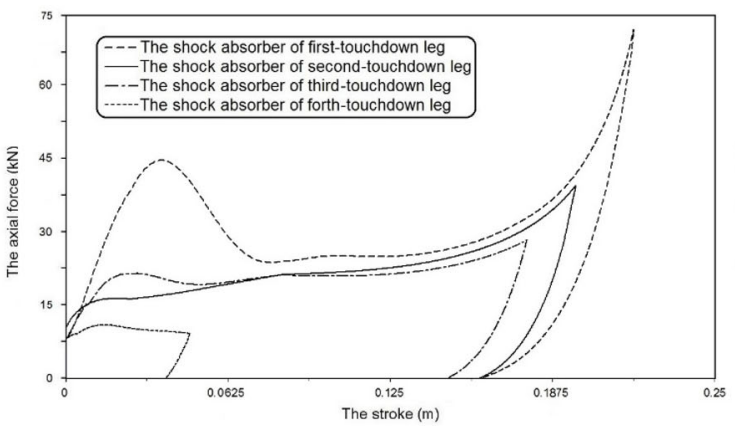

(a) Conventional struts

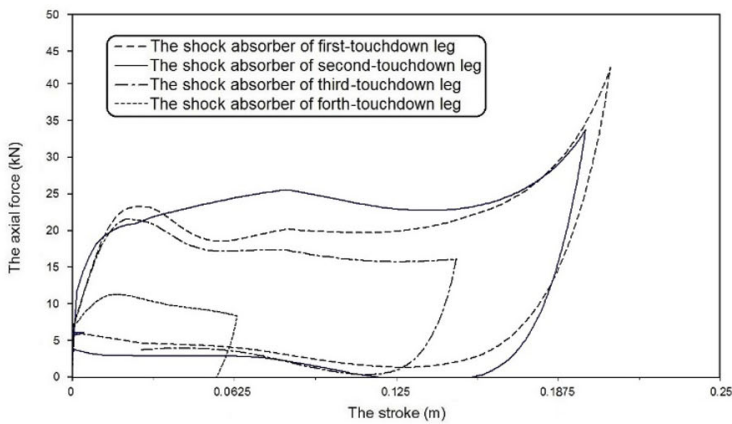

(b) Novel struts

Fig. 21 The buffer force-stroke curves of the capsule

According to the above research, the buffer efficiencies of the most compressed buffer struts are increased using the novel landing struts.

\section{Conclusions}

This paper builds a dynamic model for the rocket to analyse its modelling accuracy. Modelling accuracy is analysed by reviewing the valid methodologies for error analysis and mitigation. To meet the safety requirement under different slope landing conditions, a novel landing strut with a function of automatic adjustment is designed. Dynamic simulations are used to properly reflect the performance of the novel landing struts including the peak axial forces and buffer properties. The dynamic analysis demonstrates that:

1. The position error of the rocket $(13.46 \mathrm{~m})$ is smaller than the radius of the terminal dock of the drone ship $(25 \mathrm{~m})$; thus, the modelling accuracy of the rocket is within its accuracy requirement (with a $95 \%$ probability).

2. The rocket aims to land on a drone ship accurately and safely, whereas its payload (i.e. the capsule equipped with the novel landing struts) has enough time to adjust its struts before touchdown.

3. Compared with conventional landing struts, the novel landing struts developed in this paper have higher buffer efficiencies and shorter compression length of the most compressed buffer strut. This is because all the landing struts touch the incline terrain and absorb the landing energy at the same time.

Open Access This article is licensed under a Creative Commons Attribution 4.0 International License, which permits use, sharing, adaptation, distribution and reproduction in any medium or format, as long as you give appropriate credit to the original author(s) and the source, provide a link to the Creative Commons licence, and indicate if changes were made. The images or other third party material in this article are included in the article's Creative Commons licence, unless indicated otherwise in a credit line to the material. If material is not included in the article's Creative Commons licence and your intended use is not permitted by statutory regulation or exceeds the permitted use, you will need to obtain permission directly from the copyright holder. To view a copy of this licence, visit http://creativecomm ons.org/licenses/by/4.0/.

\section{References}

1. McNab, Ian R (2018) Electromagnetic space launch considerations. IEEE Trans Plasma Sci 2018:1-6

2. Weinzierl M (2018) Space, the final economic frontier. J Econ Perspect 32(2):173-192

3. Chang YW (2015) The first decade of commercial space tourism. Acta Astronaut 108:79-91

4. Dumont E, Sippel M (2015) Analysis of the attempt of launcher stage return by SpaceX' Falcon 9. In: Spacex workshop, Paris, Frankreich

5. Klotz I (2018) SpaceX aiming to start BFR tests next year. Aviation Week Space Technol 180(3):24-25

6. Claudia Assis (2015) Musk: SpaceX booster rocket landed 'too hard' on drone barge. Fox Business

7. Foust J (2018) SpaceX to modify Falcon 9 upper stage to test BFR technologies. Space News 29(18):6-6

8. Carpenter JD, Fisackerly R, Rosa DD et al (2012) Scientific preparations for lunar exploration with the European Lunar Lander. Planet Space Sci 74(1):208-223

9. Faraj R, Graczykowski C (2019) Hybrid prediction control for selfadaptive fluid-based shock-absorbers. J Sound Vibr 2019:5

10. Manivannan V, Langley JP, Costello MF et al (2013) Rotorcraft Slope landings with articulated landing gear. In: AIAA Paper, 2013 (2013-5160)

11. Stolz B, Brödermann T, Castiello E, Englberger G, Erne D, Gasser J, Scheuer D (2018) A novel landing gear for extending the operational range of helicopters. In: 2018 IEEE/RSJ international conference on intelligent robots and systems (IROS) (pp 1757-1763), IEEE

12. Kim D, Costello M (2016) Virtual model control of rotorcraft with articulated landing gear for shipboard landing. In: AIAA Guidance, Navigation, and Control Conference 2016, p 1863

13. Kiefer J, Ward M, Costello M (2016) Rotorcraft hard landing mitigation using robotic landing gear. J Dyn Syst Meas Control 138(3):031003

14. Ren J, Wang J, Liu X (2018) Design and Analysis of terrainadaptive bionic landing gear system. In: 2018 37th Chinese control conference (CCC) 
15. Ding Z, Wu H, Wang C et al (2019) Hierarchical optimization of landing performance for lander with adaptive landing gear. Chin J Mech Eng 32:1-12

16. Huang M (2019) Control strategy of launch vehicle and lander with adaptive landing gear for sloped landing. Acta Astronaut 161:509-523

17. Sperling FB (1964) The surveyor buffer strut. In: Proceedings of the 3rd aerospace mechanisms symposium, pp N50-17368

18. Laurenson RM, Melliere RA, Mcgehee JR (2015) Analysis of legged landers for the survivable soft landing of instrument payloads. J Spacecr Rockets 10:3

19. Sun Y, Hu Y, Liu R et al (2010) Touchdown dynamic modeling and simulation of lunar lander. In: 2010 3rd international symposium on systems and control in aeronautics and astronautics, IEEE
20. Jinbao C, Hong N, Junlin W et al (2014) Investigation on landing impact dynamic and low-gravity experiments for deep space lander. Sci China 57(010):1987-1997

21. Pham VL, Zhao J, Goo NS et al (2013) Landing stability simulation of a 1/6 lunar module with aluminum honeycomb dampers. Int $\mathbf{J}$ Aeronaut Space Sci 14(4):356-368

22. Lin Q, Kang Z, Ren J et al (2014) Impact analysis of lunar lander soft landing performance caused by the body gravity centerline shift. J Aerosp Eng 28(4):04014104

Publisher's Note Springer Nature remains neutral with regard to jurisdictional claims in published maps and institutional affiliations. 